\section{Welchen Stellenwert hat die Behandlung mit aktiviertem Vitamin D heute?}

Die meisten Nephrologen können sich eine Behandlung ihrer niereninsuffizienten Patienten ohne die Gabe von „älteren“ Vitamin-D-Analoga der ersten oder zweiten Generation nicht vorstellen. Rund $70 \%$ setzen aktivierte Vitamin-D-Analoga wie Alfacalcidol im Rahmen der medikamentösen Therapie ihrer Dialysepatienen ein, wie eine Befragung im Rahmen des Symposiums „Patient strategies 2006 and beyond - Do we need alfacalcidol anymore?" ergab. Sie vertrauen zum einen auf das Potenzial dieser Substanzen bezüglich der Kontrolle von Parathormon oder der Serumkalziumspiegel $(1,4)$. Darüber hinaus erwarten sie aber auch eine
Verbesserung der Knochenqualität oder sogar eine Verringerung des Mortalitätsrisikos der Patienten.

„Für den Nephrologen ist die Suppression der Hyperplasie der Nebenschilddrüse der wichtigste Effekt bei der Therapie mit aktiviertem Vitamin D“, konstatierte Prof. Johann D. Ringe, Leverkusen. „Doch Alfacalcidol verfügt über zusätzliche pleiotrope Effekte, die über die Suppression des Parathormons hinausgehen“, bestätigte er - angefangen bei der intestinalen Kalziumabsorption nach der Aktivierung in der Leber bis hin zur verbesserten Mineralisation der Knochenmatrix (Abb. 1, Tab. 1).

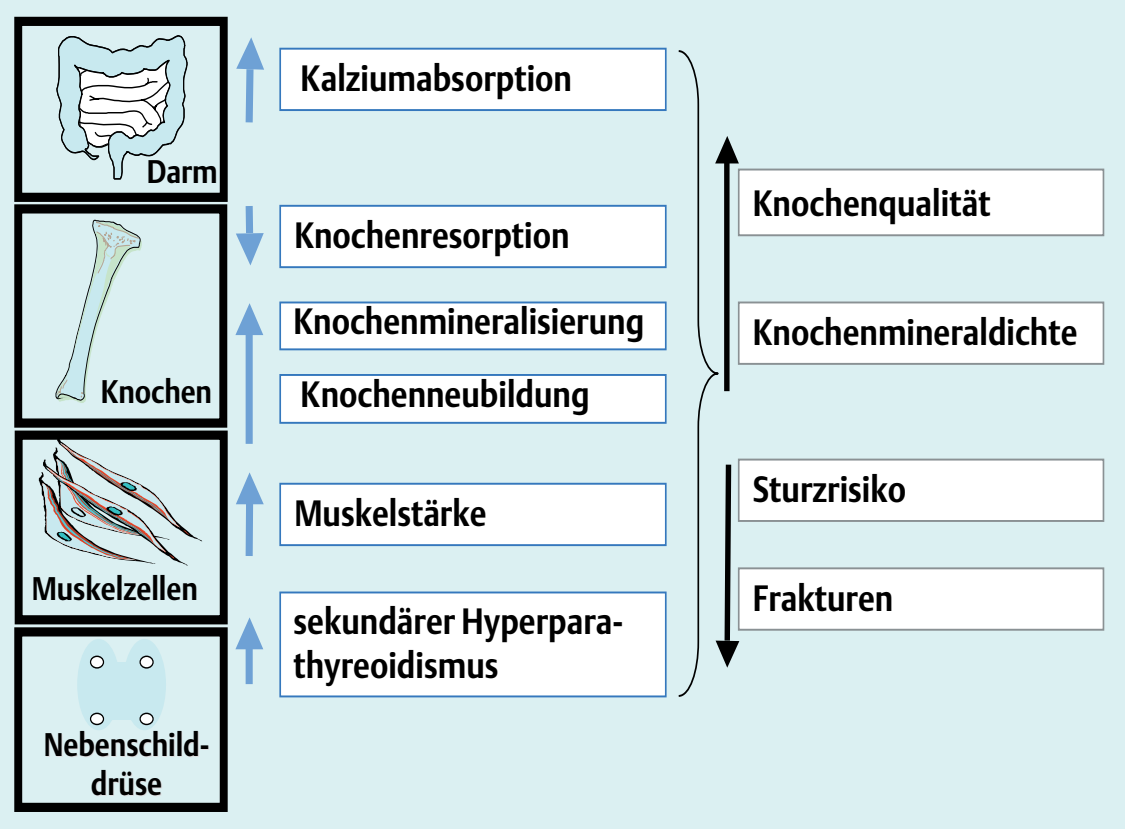

Abb. 1 Pleiotrope Effekte aktiver Vitamin- $D_{3}$-Analoga

Tab. 1 Rationale für den Einsatz von Alfacalcidol in der Osteoporosetherapie

- Herabsetzung der Parathormonausschüttung in der Nebenschilddrüse (Regulation des sekundären Hyperparathyreoidismus)

- Erhöhung der intestinalen Kalziumabsorption nach Aktivierung in der Leber

- antiresorptive Wirkung auf das Knochengewebe durch Reduktion der Osteoklasten

- Stimulation der Osteoblasten (Osteoblasten verfügen über Vitamin-D-Rezeptoren)

- verbesserte Mineralisation der Knochenmatrix und des neu gebildeten Knochengewebes

- positive Wirkungen auf den Muskelstoffwechsel und die neuromuskuläre Koordination (Muskeln verfügen über Vitamin-D-Rezeptoren)

- immunsuppressive, immunmodulierende und antiresorptive Wirkungen
Knochen und Muskeln beeinflussen das Frakturrisiko

Knochenbezogene und muskuläre Wirkungen bilden eine zusätzliche Rationale für den Einsatz von aktiviertem Vitamin $\mathrm{D}$ bei der Prävention und Behandlung metabolischer Knochenerkrankungen (Osteomalazie oder renale Knochenerkrankungen) und der primären oder sekundären Osteoporose. Während die positiven Effekte einer Therapie mit Alfacalcidol auf Knochendichte und -qualität in der Regel bekannt sind, wird die Rolle des Muskelkompartiments bei der Pathogenese einer osteoporotischen Fraktur häufig unterschätzt. „Doch es ist ganz einfach“, meinte Ringe. Sind Muskelmasse und -funktion beeinträchtigt, bewegen sich die Patienten seltener, es verringert sich die Knochendichte, woraus ein erhöhtes Osteopenierisiko und damit ein erhöhtes Frakturrisiko resultiert. Zudem steigt natürlich das Sturz- und Traumarisiko, wenn die Muskulatur nur schwach ausgebildet ist.

„Es ist belegt, dass Muskel- und Nervenzellen Vitamin-D-Rezeptoren beinhalten, deren Zahl jedoch mit zunehmendem Alter abnimmt“, berichtete Ringe. „Es reicht aber nicht aus, natives Vitamin D zu supplementieren. Nur mit aktivierten Vitamin-D-Analoga kann man diesem Ungleichgewicht gegensteuern“, erklärte er. Zum Beispiel könne eine Alfacalcidoltherapie den Anteil der schnellen Typ-2-Muskelfasern erhöhen (6).

\section{Frakturrisiko deutlich senken}

Tatsächlich lässt sich mit einer Gabe von Alfacalcidol das Sturzrisiko um 55\% und damit auch die Zahl der Frakturen signifikant reduzieren, berichtete Ringe - allerdings nur, wenn eine Basisversorgung mit Kalzium gewährleistet ist (2). Im Gegensatz zu Cholecalciferol, das bei älteren Patienten oftmals nicht aktiviert werden kann, ist Alfacalcidol eine effektive Therapie mit dem Prodrug des Vitamin-D-Hormons, die bei Osteoporosepatienten zu einer deutlichen Reduktion des Frakturrisikos führt (3).

Eine noch effektivere Therapie ist durch eine zusätzliche Alendronatbehandlung möglich, wie jetzt die Zwei-Jahres-Ergebnisse der ACC1-Studie zeigen. Hier war sowohl bei Patientinnen mit postme-

${ }^{1}$ Alfacalcidol-Alendronate Combined 
nopausaler Osteoporose als auch bei Männern mit Osteoporose $(\mathrm{n}=90)$ - der Zuwachs der Knochenmineraldichte am stärksten ausgeprägt, wenn die Studienteilnehmer sowohl Alfacalcidol als auch Alendronat einnahmen. Auch die Zahl der Stürze ging unter der Kombinationstherapie signifikant zurück. Dementsprechend waren unter der Zweifachtherapie deutlich weniger neue Frakturen zu verzeichnen, berichtete Ringe.

\section{K-DOQI-Zielwerteinstellung ist keine leichte Aufgabe}

Störungen im Knochenstoffwechsel sind jedoch nur ein Teil des vielteiligen Puzzles, dem sich der behandelnde Arzt bei der Therapie seiner Patienten mit einer fortgeschrittenen chronischen Nierenerkrankung (Stadium III-V) gegenübersieht. Die bestehenden Dysbalancen im Mineralstoffwechsel sind auch mit einer höheren kardiovaskulären und renalen Morbidität und Mortalität assoziiert, erinnerte Prof. Preben Joffe, Odense (Dänemark). Daher ist es von essenzieller Bedeutung, die Patienten stabil auf die Zielwerte der aktuellen K/DOQI-Leitlinien einzustellen. Doch dies sei nicht ganz leicht, denn die Therapieziele sind verschiedenen Limitationen unterworfen.

\section{Kontrolle der Phosphat- und}

Kalziumspiegel bei Dialysepatienten

Der erste Schritt ist die Kontrolle einer bestehenden Hyperphosphatämie. Eine dietäre Phosphatrestriktion ist bei den Patienten oft nur schwer durchzusetzen. „Mit der strikten Einhaltung einer phosphat- und damit auch eiweißarmen Diät nehmen Sie dem Patienten oft den letzten verbliebenen Rest seiner Lebensqualität das Essen“, konstatierte Joffe. Daher werden wohl nur wenige Patienten über längere Zeit diese strengen Diätvorschriften befolgen.

„Und wenn sich die Patienten daran halten, kommt es leicht zu einer Malnutrition“, warnte Joffe. Denn eine restriktive Eiweißreduktion ist in der Regel mit einer verringerten Gesamtkalorienaufnahme assoziiert. Ein solcher Eiweiß- und Kalorienmangel führt aber zu einer katabolen Stoffwechsellage. Dies wiederum hat schnell eine geringere physikalische Aktivität der Patienten und im Extremfall eine erhöhte Mortalität zur Folge.

Ein Ausweg aus diesem Konflikt ist die medikamentöse Therapie der Hyperphosphatämie. Doch Vorsicht: Kalzium- haltige Phosphatbinder tragen zusätzlich zu der zum Teil hohen Kalziumbelastung über das Dialysat zu einem zweiten Dilemma bei. Hohe Kalziumspitzenwerte können eine Hyperkalziämie bedingen, die als eine der Ursachen für eine Kalzifizierung der Gefäße und damit als Ursache kardiovaskulärer Erkrankungen gilt.

\section{Kontrolle des Parathormons}

mit Vitamin D

Obwohl eine Therapie mit aktiviertem Vitamin $\mathrm{D}_{3}$ zur Prävention eines sekundären Hyperparathyreoidismus indiziert ist, sind viele Dialysepatienten aus Furcht vor den potenziell möglichen kalzämischen Wirkungen nicht ausreichend damit supplementiert, erklärte Joffe. Doch damit verliere man nicht nur die wichtigen pleiotropen Effekte des Vitamin- $\mathrm{D}_{3}-$ Hormons. Zudem induziere man unter Umständen eine Hyperkalzämie, die wiederum das kardiovaskuläre Risiko der Patienten deutlich erhöhe.

Dass sich dies durchaus auch auf das Mortalitätsrisiko der Patienten auswirkt, belegt die vor zwei Jahren veröffentlichte Kohortenstudie von Teng et al. (7). Demnach sinkt nicht nur die kardiovaskuläre Mortalität der Patienten, denen aktives Vitamin D infundiert wurde, hochsignifikant von 14,6 auf 7,6\%. Auch die Gesamtmortalität der Patienten reduzierte sich unter der i.v.-Therapie mit aktiviertem Vitamin D drastisch: Ohne der i.v.-Gabe von aktiviertem Vitamin D betrug die ZweiJahres-Mortalitätsrate pro 100 Personenjahre $28,6 \%$. Unter aktiviertem Vitamin D (i.v.) dagegen sank die Rate auf 13,8\% $(\mathrm{p}<0,001)$.

Allerdings kann eine Therapie mit aktiven Vitamin-D-Metaboliten in Verbindung mit erhöhten Parathormonspiegeln unter Umständen auch eine Hypokalzämie induzieren. Auch hier ist die erhöhte Parathormonproduktion eine der Ursachen für eine verstärkte Mobilisation von Kalzium aus dem Knochen und damit ein langfristig erhöhtes kardiovaskuläres Risiko. Doch unter einer Therapie mit Alfacalcidol verstarben in einer plazebokontrollierten Untersuchung weniger Patienten an kardiovaskulären Komplikationen, wenn sie eine Alfacalcidoltherapie erhielten $(p=0,013 ; 5)$.

Neue Therapieoptionen sind derzeit schlicht zu teuer

Hohe Kosten und die nicht vorhersehbaren Langzeiteffekte sind die Crux neuer
Therapieoptionen, wie kalziumfreie Phosphatbinder oder Calcimimetika. „Zwar glaube ich, dass zum Beispiel Calcimimetika eine wichtige neue Option zur Kontrolle des Knochen- und Mineralstoffwechsels sind“, meinte Joffe. Sie haben jedoch Nachteile wie einen signifikanten Abfall des Testosteronspiegels. Außerdem wies Joffe auf Unsicherheiten ihres Einsatzes bei Patienten mit eingeschränkter Leberfunktion und mögliche Nebenwirkungen wie Übelkeit und Erbrechen hin. Darüber hinaus verwies Joffe auf die schwierige Kostensituation: „Sogar in einem vergleichsweise so reichen europäischen Land wie Dänemark, ist es mir nicht gestattet, Calcimimetika einzusetzen."

Und was ist mit den neuen aktivierten Vitamin-D-Analoga wie Paricalcitol? „Ich kann derzeit keine pharmakokinetischen Vorteile dieser Substanzen im Vergleich zu Alfacalcidol erkennen“, sagte Joffe. Die umfangreichen pleiotropen Effekte seien bislang zwar für Calcitriol und Alfacalcidol, nicht aber für das Paricalcitol beschrieben. Der Nephrologe forderte daher direkte Vergleichsstudien zwischen den Substanzen.

„In meinen Augen haben die älteren aktivierten Vitamin- $\mathrm{D}_{3}$-Analoga wie Alfacalcidol noch immer eine Schlüsselrolle bei der Behandlung von chronisch nierenkranken Patienten“, schloss Joffe - einem Statement, dem sich in der abschließenden interaktiven Abstimmung gut $80 \%$ der Teilnehmer des Symposiums anschlossen. Sie bewerteten Alfacalcidol als aktive Therapieoption (17,3\%), sehen ihren Vorteil in den pleiotropen Effekten (4,9\%) - oder beides (61,7\%)!

sts

Literatur

1 Brandi L et al. Am J Nephrol 1992; 12 (5): 311-218

2 Dukas LC et al. J Am Geriatr Soc 2004; 52 (2): 230-236

3 Francis RM et al. Osteoporos Int 1996; 6 (4): 284-290

4 Moriniere P et al. Kidney Int 1993; 43 (suppl 41): S121-S124

5 Shoji T et al. Nephrol Dial Transplant 2004; 19 (1): 179-184

6 Sorenson OH et al. Clin Sci (Lond) 1979; 56 (2): 157-161

7 Teng M et al. J Am Soc Nephrol 2005; 16 (4): 1115-1125

Quelle: Symposium „Patient strategies 2006 and beyond - Do we need alfacalcidol anymore?" im Rahmen des ISN Nexus, veranstaltet von LEO Pharma

Dieser Artikel entstand mit freundlicher Unterstützung der LEO Pharma GmbH, Neu Isenburg 